Check for updates

Cite this: RSC Adv., 2018, 8, 21002

\title{
Chemical studies on the parasitic plant Thonningia sanguinea Vahl $\uparrow$
}

\author{
Ama Kyeraa Thomford, (DD ab Reda Fouad Ahmed Abdelhameed ${ }^{\text {ac }}$ and Koji Yamada*a
}

Thonningia sanguinea Vahl plays an important role in traditional medicine in many African cultures. A study of the $n$-hexane fraction of the whole plant of $T$. sanguinea led to the isolation of two glucocerebroside molecular species TSC-1 and TSC-2, one $\beta$-sitosteryl-3 $\beta$-D-glucopyranoside- $6^{\prime}$-O-fatty acid ester molecular species, TSS-1, and seven known triterpenes (1-7). The ethyl acetate fraction also afforded five known lignans (8-12) and one known flavanone (13). Their structures were elucidated by means of chemical and spectroscopic methods (methanolysis, NMR and mass spectrometry). Spectral analyses of the glucocerebrosides revealed mainly sphingosine-type (TSC-1) and phytosphingosine-type (TSC-2) cerebrosides, with both possessing mainly 2-hydroxy fatty acid and $\beta$-D-glucopyranose moieties. TSS-1 was found to be a $\beta$-sitosterol-type with fatty acid methyl esters and $\beta$-D-glucopyranoside moieties. The classes of compounds isolated from this plant are well known for their interesting biological activities including antimicrobial, antioxidant, anticancer, antiinflammatory and analgesic. They may therefore be responsible in part or in whole for these activities, hence validating the traditional uses of the plant. To the best of our knowledge, this is the first report on the isolation of all these compounds from $T$. sanguinea.

Received 7th May 2018

Accepted 1st June 2018

DOI: $10.1039 / \mathrm{c} 8 \mathrm{ra03913e}$

rsc.li/rsc-advances sometimes for prophylaxis, ${ }^{2}$ sexually transmitted diseases and as an aphrodisiac. It is used to treat diarrhoea and worm infestation in Cote d'Ivoire and Congo. It is also mixed with Capsicum to produce a topical cream for treating haemorrhoids and torticollis. It is also used to treat dysentery, sore throat, skin infections, abscesses, dental caries, gingivitis, fever, malaria, heart disease, rickets, and rheumatism. ${ }^{\mathbf{1 , 3} 4}$

Chemically, an aqueous and hydroalcoholic flower extract of T. sanguinea revealed the presence of alkaloids, catechin tannins, flavonoids, saponins, quinones and polyphenols on preliminary screening. ${ }^{5}$ Brevifolin carboxylic acid (BCA), ${ }^{6}$ gallic acid (GA) ${ }^{6}$ and two ellagitannins: thonningianins $\mathrm{A}$ (Th A) and Th $\mathrm{B}^{7}$ are the only four compounds reported to have been isolated from the plant. N'guessan et al. reported that both GA and BCA, isolated from $T$. sanguinea demonstrated moderate antibacterial activity against Salmonella enteritidis, Salmonella typhimurium, and Salmonella abony in the disc diffusion method, with significant antioxidant activity in the DPPH radical scavenging activity assay. ${ }^{6} \mathrm{GA}$ is well known as a potent antioxidant phenolic compound, with numerous biological activities including antitumor, antimicrobial and antimelanogenic. ${ }^{6}$ BCA has been shown to inactivate HBsAg and inhibit hepatitis B virus replication and tumour growth. ${ }^{8}$ The ellagitannins isolated from the plant also possessed hepatoprotective actions, potent antimicrobial effects ${ }^{\mathbf{4 , 6 , 7 , 9}}$ and significant free radical scavenging activity against DPPH by ESR analysis. ${ }^{7}$ Th A effectively inhibited of the proliferation of HepG2 human hepatocellular carcinoma cells by inducing apoptosis. This was observed as an increase in the sub-G1 cell population, 
DNA fragmentation, and increase in the content of reactive oxygen species. ${ }^{10} \mathrm{Th}$ A was also shown to be a potent in vitro inhibitor of rat liver crude glutathione S-transferases (GSTs) and hGSTP1-1 activity. ${ }^{11}$

Biological study on T. sanguinea plant extracts (in vivo and in vitro assays) indicated the prophylactic potential of the aqueous extract and its $n$-butanolic fraction in bronchial asthma. The active agents extracted into $n$-butanol and may be flavonoids and/or phenolic in nature. ${ }^{12}$ The anticoccidial activity of the extract against Eimeria sp. sporazoites, ${ }^{13}$ antibacterial effects against some multidrug resistant strains of Salmonella enterica ${ }^{16}$ and their effects on extended spectrum- $\beta$-Lactamases (ESBL) producing Escherichia coli and Klebsiella pneumoniae strains ${ }^{3}$ have been reported. The significant antimalarial effects of $T$. sanguinea root extracts against Plasmodium falciparum (in vitro), ${ }^{14}$ Plasmodium berghei and Plasmodium chabaudi (in vivo) ${ }^{1}$ have also been reported. The aqueous extract of $T$. sanguinea exhibited hepatoprotective activity towards a variety of different toxicants including galactosamine, carbon tetrachloride and aflatoxin B1. ${ }^{2,15}$ It also suppressed CYP3A2 and CYP1A2 expression at the level of transcription, ${ }^{16}$ protects against aflatoxin B1 acute hepatotoxicity in Fischer 344 rats $^{2}$ and also inhibits the liver drug metabolising enzymes of rats. ${ }^{17}$

The aqueous decoction of $T$. sanguinea has been used for more than thirty five years as a mono-herbal product produced by the Centre for Plant medicine Research, AkuapemMampong, Ghana (CPMR) under the registered names CAMPA-T ${ }^{\circledR}$ and NINGER ${ }^{\circledR}$. Due to the reported clinical effectiveness of these herbal formulations at the CPMR clinic, they have been approved for use as standardised herbal medicines by the Food and Drug Authority. CAMPA-T® and NINGER ${ }^{\circledR}$ are now part of the Essential Herbal Medicines List recommended for use in pilot herbal clinics under the Integrated Herbal Medicine Services Programme in public hospitals. These $T$. sanguinea-based products are prescribed for the management of arthritic pain, sexual weakness, male and female infertility, dysmenorrhoea, amenorrhoea and uterine fibroids. Therefore, $T$. sanguinea is an important medicinal plant that is contributing immensely to public health care in Ghana.

Despite the interesting clinical usage in Ghana, as well as the reported pharmacological activities of the extracts of $T$. sanguinea, there is no detailed study on the chemical composition of the plant. In this report, we seek to provide a chemical profile for $T$. sanguinea to better understand these reported pharmacological activities and give credence to its use in traditional medicine.

\section{Experimental}

\subsection{General experimental information}

NMR spectra were recorded in chloroform- $d$, methanol- $d_{4}$ and pyridine- $d_{5}$ (Nacalai Tesque, Inc., Kyoto, Japan) with Varian Unity Plus 400 spectrometer (Palo Alto, CA, USA) operating at $400 \mathrm{MHz}$ for ${ }^{1} \mathrm{H}$ and $100 \mathrm{MHz}$ for ${ }^{13} \mathrm{C}$, and with a JEOL JNM-AL 300 spectrometer (JEOL Ltd, Tokyo, Japan) at $300 \mathrm{MHz}$ for ${ }^{1} \mathrm{H}$ NMR. The UV spectra were recorded using a double beam Shimadzu UV-visible spectrophotometer (model UV-1601 PC, Kyoto
City, Japan). IR spectra were recorded using a Jasco FT/IR-410 K spectrometer (Jasco Co. Ltd., Tokyo, Japan) with a range of 400$4000 \mathrm{~cm}^{-1}$. FAB-MS spectra were recorded on a JMS $700 \mathrm{~N}$ spectrometer (JEOL Ltd., Tokyo, Japan) in positive ion mode, with glycerol or $m$-nitrobenzyl alcohol, with or without $\mathrm{NaCl}$, as the matrix. The optical rotation measurements were done using a Jasco P-1020 polarimeter (Jasco Co. Ltd., Tokyo, Japan). Extraction and isolation of compounds were done the following solvents: acetone, acetonitrile, $n$-butanol, chloroform, ethyl acetate, $n$-hexane and methanol (Nacalai Tesque, Inc., Kyoto, Japan). Column chromatography (CC) was performed using Sephadex LH-20 (25-100 mm, GE Healthcare UK Ltd., Buckinghamshire HP7 9NA, UK), silica gel Purasil $60 \AA$, 230-400 mesh (Whatman, Sanford, ME, USA) and Cosmosil $140 \mathrm{C}_{18^{-}}$ PREP silica gel $90 \AA$ A 40-63 mesh (Nacalai Tesque, Inc., Kyoto, Japan). TLC was performed on $0.25 \mathrm{~mm}$ thick, precoated silica gel $60 \mathrm{~F}_{254}$ and silica gel RP-18 $\mathrm{F}_{254}$ plates (Merck, Darmstadt, Germany). Prep. TLC was performed on $2 \mathrm{~mm}$ thick PLC silica gel $60 \mathrm{~F}_{254}$ glass plates (Merck, Darmstadt, Germany). Spots were developed with $5 \% \mathrm{H}_{2} \mathrm{SO}_{4}: \mathrm{MeOH}$ and detected by illumination under a short wavelength UV $(254 \mathrm{~nm})$. Analytical HPLC was performed on a Cosmosil $5 \mathrm{C}_{18}$-AR-II $4.6 \mathrm{~mm} \times$ $250 \mathrm{~mm}$ column (Nacalai Tesque, Inc., Kyoto, Japan) with methanol (Nacalai Tesque, Inc., Kyoto, Japan) at a flow rate of $0.8 \mathrm{~mL} \mathrm{~min}{ }^{-1}$ and Cosmosil-sugar-D $4.6 \mathrm{ID} \times 250 \mathrm{~mm}, 1$ $\mathrm{mL} \min ^{-1}$, refractive index (RI) detector using $95 \%$ acetonitrile. Preparative HPLC was performed on a Develosil $5 \mathrm{C}_{18} 4.6 \mathrm{~mm} \times$ $150 \mathrm{~mm}$ column (Nacalai Tesque, Inc., Kyoto, Japan) using $100 \% \mathrm{MeOH}$ as solvent, at a flow rate of $0.5 \mathrm{~mL} \mathrm{~min}^{-1}$ on a Jasco DG-2080-53 Plus degasser, Jasco PU-2080 Plus pump, Jasco AS2055 Plus auto sampler, Jasco CO-2065 Plus column oven (maintained at $35{ }^{\circ} \mathrm{C}$ ) and Jasco MD-2018 Plus PDA detector (Jasco Co. Ltd., Tokyo, Japan).

\subsection{Plant collection and identification}

Thonningia sanguinea whole plant was collected by the staff of the Centre for Plant Medicine Research from the eastern region of Ghana in the month of January, 2015 and authenticated by the curator of their herbarium. A voucher specimen with the number CSRPM no. 140 was assigned to the sample.

\subsection{Extraction and isolation}

The whole plant of $T$. sanguinea was shade-dried for seven days and pulverised. The dried powdered plant material $(3.5 \mathrm{~kg})$ was extracted by cold maceration with $\mathrm{MeOH}(3 \times 10 \mathrm{~L}$ for 3 days $)$, followed by $\mathrm{MeOH}: \mathrm{CHCl}_{3}(1: 1 ; 3 \times 10 \mathrm{~L}$ for 3 days). The filtrates were pooled together and concentrated in vacuo using the rotary evaporator. The methanol/chloroform crude extract $(423 \mathrm{~g})$ was dissolved in distilled water and serially partitioned between $n$-hexane, ethyl acetate, $n$-butanol solvents to obtain the $n$-hexane (20 g), ethyl acetate (260 g), $n$-butanol (88 g) and aqueous $(30 \mathrm{~g})$ fractions. The $n$-hexane fraction $(20 \mathrm{~g})$ was subjected to silica gel CC $(800 \mathrm{~g})$ using $n$-hexane : EtOAc $(9: 1$ $1: 9), \mathrm{CHCl}_{3}: \mathrm{MeOH}(8: 2-6: 4)$ and $80 \%$ acetone to give sixteen sub-fractions (H-1-H-16). Fraction H-4 $(500 \mathrm{mg})$ was subjected to repeated silica gel CC using $n$-hexane : EtOAc 
(8.5: 1.5-1:1) to afford compound 6 (200 mg). Fraction $\mathrm{H}-5$ (330 mg) was subjected to repeated silica gel $\mathrm{CC}$ using $\mathrm{CHCl}_{3}$ : MeOH (1:0-1:1) to afford seven fractions. H-5-6 (138 mg) was further chromatographed on a $\mathrm{C}_{18} \mathrm{RP}$ silica gel column (10 g) using $\mathrm{MeOH}: \mathrm{H}_{2} \mathrm{O}(8: 2-1: 0)$ and acetone : $\mathrm{H}_{2} \mathrm{O}(1: 1-1: 0)$ to afford compound 7 (22 mg). Fraction $\mathrm{H}-10$ (600 mg) was chromatographed on silica gel $(20 \mathrm{~g})$ using $\mathrm{CHCl}_{3}: \mathrm{MeOH}$ (9.8:0.2-1:1) to afford eleven sub-fractions ( $\mathrm{H}-10-1-\mathrm{H}-10-11)$. $\mathrm{H}-10-4$ (350 $\mathrm{mg}$ ) was further chromatographed on a $\mathrm{C}_{18} \mathrm{RP}$ silica gel column (10 g) using $100 \% \mathrm{MeOH}$ to afford compound 1 (28 mg), TSS-1 (94 mg) and a mixture purified by preparative HPLC using $100 \% \mathrm{MeOH}$ to afford compound $2(40 \mathrm{mg})$ and 4 (15 mg). Fraction H-13 (2.3 g) was chromatographed on silica gel $(100 \mathrm{~g})$ using $\mathrm{CHCl}_{3}: \mathrm{MeOH}(9.8: 0.2-2: 8)$ to afford five subfractions $\mathrm{H}-13-1-\mathrm{H}-13-5$. $\mathrm{H}-13-4$ (340 $\mathrm{mg}$ ) was subjected to repeated silica gel CC using $\mathrm{CHCl}_{3}: \mathrm{MeOH}(9.5: 0.5-1: 1)$ to afford TSC-1 (30 mg), TSC-2 (45 mg) and a mixture purified by preparative HPLC using $100 \% \mathrm{MeOH}$ to afford compound 3 (13 $\mathrm{mg})$ and 5 ( $8 \mathrm{mg})$. The ethyl acetate fraction $(70 \mathrm{~g})$ was subjected to silica gel CC (240 g) using $\mathrm{CHCl}_{3}: \mathrm{MeOH}(9.8: 0.2-3: 7)$ and $80 \%$ acetone to afford twenty two sub-fractions (E-1-E-22). Fraction E-2 (1.08 g) was subjected to silica gel CC (10 g) using $n$-hexane : EtOAc $(8: 2-2: 8)$ to give fourteen subfractions (E-2-1-E-2-14) which afforded compound 8 (100 mg) and 9 (200 mg). Fraction E-9 (500 mg) was subjected to silica gel CC (15 g) using $\mathrm{CHCl}_{3}: \mathrm{MeOH}(9.9: 0.1-9.6: 0.4)$ to give sixteen sub-fractions (E-9-1-E-9-16). E-9-7 (175 mg) was subjected to repeated silica gel CC $(10 \mathrm{~g})$ using $n$-hexane : EtOAc $(8: 2-1: 1)$ and $\mathrm{CHCl}_{3}: \mathrm{MeOH}(9.7: 0.3-1: 1)$ to afford compound 10 (14 mg) and 11 (23 mg). E-9-8 (168 mg) was chromatographed on a $\mathrm{C}_{18} \mathrm{RP}$ silica gel column $(10 \mathrm{~g})$ using $\mathrm{MeOH}: \mathrm{H}_{2} \mathrm{O}(3: 7-4: 6)$ to afford compound $12(117 \mathrm{mg})$ and 13 (18 mg).

2.3.1 TSS-1. White amorphous powder. IR $\nu_{\max } / \mathrm{cm}^{-1}: 3350$ (hydroxy), 1720 (carbonyl). Positive-ion FAB-MS: $m / z$ 837, 851, $865,879,893,806,907,921,935,949,963$ and $977[\mathrm{M}+\mathrm{Na}]^{+}$ series. ${ }^{1} \mathrm{H}$ NMR $\left(\mathrm{CDCl}_{3}\right) \delta_{\mathrm{H}}: 0.68-0.93(3 \mathrm{H}, \mathrm{t}), 1.25\left(2 \mathrm{H}, \mathrm{s}, n \mathrm{CH}_{2}\right)$, $4.37\left(1 \mathrm{H}, \mathrm{d}, J=8.0 \mathrm{~Hz}\right.$, glucose $\left.\mathrm{H}-1^{\prime}\right) .{ }^{13} \mathrm{C}$ NMR: see Spectral data.

2.3.2. TSC-1. White amorphous powder. IR $\nu_{\max } / \mathrm{cm}^{-1}: 3422$ (hydroxy), 1640 and 1540 (amide). $[\alpha]_{\mathrm{D}}^{20}=-31.3$ ( $c 0.1$ in $\mathrm{MeOH}$ ). Positive-ion FAB-MS: $m / z$ 736, 764, 778, 792, 806, 820, 834, 848 and $862[\mathrm{M}+\mathrm{Na}]^{+}$series. ${ }^{1} \mathrm{H}$ NMR $\left(\mathrm{C}_{5} \mathrm{D}_{5} \mathrm{~N}\right) \delta_{\mathrm{H}}: 0.86(6 \mathrm{H}$, br. t $)$, $4.89\left(1 \mathrm{H}, \mathrm{d}, J=8.0 \mathrm{~Hz}\right.$, glucose $\left.\mathrm{H}-1^{\prime \prime}\right) .{ }^{13} \mathrm{C}$ NMR: see Table 1.

2.3.3 TSC-2. White amorphous powder. IR $\nu_{\max } / \mathrm{cm}^{-1}: 3289$ (hydroxy), 1640, 1540 (amide). $[\alpha]_{\mathrm{D}}^{22}=+29$ (c 0.1 in $\mathrm{MeOH}$ ). Positive-ion FAB-MS: $m / z$ 810, 824, 838, 852, 866 and $880[\mathrm{M}+$ $\mathrm{Na}]^{+}$series. ${ }^{1} \mathrm{H}$ NMR $\left(\mathrm{C}_{5} \mathrm{D}_{5} \mathrm{~N}\right) \delta_{\mathrm{H}}: 0.83(6 \mathrm{H}$, br. t), $4.93(1 \mathrm{H}, \mathrm{d}, J=$ $8.0 \mathrm{~Hz}$, glucose $\left.\mathrm{H}-1^{\prime \prime}\right) .{ }^{13} \mathrm{C}$ NMR: see Table 1 .

2.3.4 Methanolysis of TSC-1. TSC-1 $(5 \mathrm{mg})$ was heated with $5 \% \mathrm{HCl}$ in $\mathrm{MeOH}(0.5 \mathrm{~mL})$ at $70{ }^{\circ} \mathrm{C}$ for $8 \mathrm{~h}$ in a sealed smallvolume vial. The reaction mixture was extracted with $n$ hexane and the extract was concentrated in vacuo to yield a mixture of fatty acid methyl ester (FAME) products. The methanolic layer was neutralized with $\mathrm{Ag}_{2} \mathrm{CO}_{3}$, filtered, and the filtrate was concentrated in vacuo to give a mixture of long chain base (LCB) and methyl glycoside products. The mixture was further evaporated and reacted with acetic anhydride/ pyridine $(1: 1)(0.2 \mathrm{~mL})$ at $70{ }^{\circ} \mathrm{C}$ for $8 \mathrm{~h}$ in a sealed smallvolume vial followed by evaporation in vacuo to dryness. The mixture was separated using preparative TLC to afford the LCB, LCB acetates and LCB glucoacetates. ${ }^{1} \mathrm{H},{ }^{13} \mathrm{C}$ NMR and FAB-MS analyses were performed on the FAMEs and the LCB products.

2.3.5 Methanolysis of TSC-2. In the same manner as described for TSC-1, TSC-2 (5 mg) was methanolyzed and the reaction mixture was worked up to give the FAMEs, LCB, LCB acetates and LCB glucoacetates. The ${ }^{1} \mathrm{H},{ }^{13} \mathrm{C}$ NMR and FAB-MS analyses were performed on the FAMEs and the LCB products.

2.3.6 FAB-MS analysis of the FAME mixture from TSC-1. Positive molecular ion peaks at 287, 315, 329, 343, 357, 371, 385, 399 and $413[\mathrm{M}+\mathrm{H}]^{+}$indicated the presence of $\mathrm{C}-16, \mathrm{C}-18-\mathrm{C}-25$ fatty acid methyl esters in TSC-1. See Fig. 1.

2.3.7 FAB-MS analysis of the LCB products from TSC-1. Positive ion FAB-MS analysis showed molecular ion peaks at 395 $[\mathrm{M}+](\mathrm{LCB})$ and $424[\mathrm{M}+\mathrm{H}]^{+}$(LCB acetate) indicating the presence of a C-16 LCB, while $652[\mathrm{M}+\mathrm{Na}]^{+}$(LCB glucoacetate) indicated the presence of a C-18 LCB in TSC-1. See Fig. 1.

2.3.8 FAB-MS analysis of the FAME mixture from TSC-2. Positive molecular ion peaks at 343, 357, 371, 385, 399 and 413 $[\mathrm{M}+\mathrm{H}]^{+}$indicated the presence of C-20-C-25 fatty acid methyl esters in TSC-2. See Fig. 1.

2.3.9 FAB-MS analysis of the LCB products from TSC-2. Positive ion FAB-MS analysis showed molecular ion peaks at 455 $[\mathrm{M}+]\left(\mathrm{LCB}\right.$ acetate) and $484[\mathrm{M}+\mathrm{H}]^{+}$(LCB acetate) indicating the presence of a C-16 and C-18 LCB respectively in TSC-2. See Fig. 1.

2.3.10 Identification of the sugar moiety in TSC-1. TSC-1 $(5 \mathrm{mg})$ was heated with $5 \% \mathrm{HCl}$ in $\mathrm{MeOH}(0.5 \mathrm{~mL})$ at $70{ }^{\circ} \mathrm{C}$ for $8 \mathrm{~h}$ in a sealed small-volume vial. The reaction mixture was extracted with $\mathrm{CHCl}_{3}$ to remove the release fatty acid. The methanolic was neutralized with $\mathrm{Ag}_{2} \mathrm{CO}_{3}$ to give the methylated sugar followed by HPLC analysis HPLC analysis

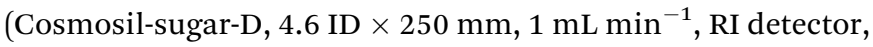
$95 \%$ acetonitrile) against standard glucose and galactose. TSC1 showed a retention time identical to glucose (glucose $t_{\mathrm{R}}=$ $14.11 \mathrm{~min}$, galactose $\left.t_{\mathrm{R}}=13.27 \mathrm{~min}\right)$. In the same way, the sugar moiety was identified as glucose for TSC-2 and TSS-1.

2.3.11 Determination of the absolute configuration of the glucose moiety in TSC-1 (Tanaka et al. Method). ${ }^{18}$ The glycosidic bond in TSC-1 $\left(2 \mathrm{mg}, 1.1 \times 10^{6} \mathrm{~mol}\right)$ was hydrolyzed by heating in $0.5 \mathrm{M} \mathrm{HCl}(0.1 \mathrm{~mL})$ and neutralized with Amberlite IRA400. After drying in vacuo, the residue was dissolved in pyridine $(0.1 \mathrm{~mL})$ containing L-cysteine methyl ester hydrochloride $(0.5 \mathrm{mg})$ and heated at $60{ }^{\circ} \mathrm{C}$ for $1 \mathrm{~h}$. A $0.1 \mathrm{~mL}$ solution of $o$-tolyl isothiocyanate $(0.5 \mathrm{mg})$ in pyridine was added to the mixture, which was heated at $60{ }^{\circ} \mathrm{C}$ for $1 \mathrm{~h}$. The reaction mixture was directly analyzed by reversed-phase HPLC. The peaks at 18.68 min which were coincided with the arylisothiocyanate derivative of $\mathrm{D}$-glucose ( $\mathrm{L}$-glucose $t_{\mathrm{R}}=19.22$ min). In the same way as described for TSC-1, the absolute configuration of the glucose moiety (D-form) of TSC-2 (at $t_{\mathrm{R}}=$ $18.5 \mathrm{~min}$ ) and TSS-1 (at $t_{\mathrm{R}}=18.7 \mathrm{~min}$ ) were also determined. See Fig. 1 and 2; Spectral data. 
Table $1{ }^{1} \mathrm{H}$ and ${ }^{13} \mathrm{C}$ NMR spectral data of TSC-1 and TSC-2 (measured in $\mathrm{C}_{5} \mathrm{D}_{5} \mathrm{~N}$ ) ${ }^{a}$

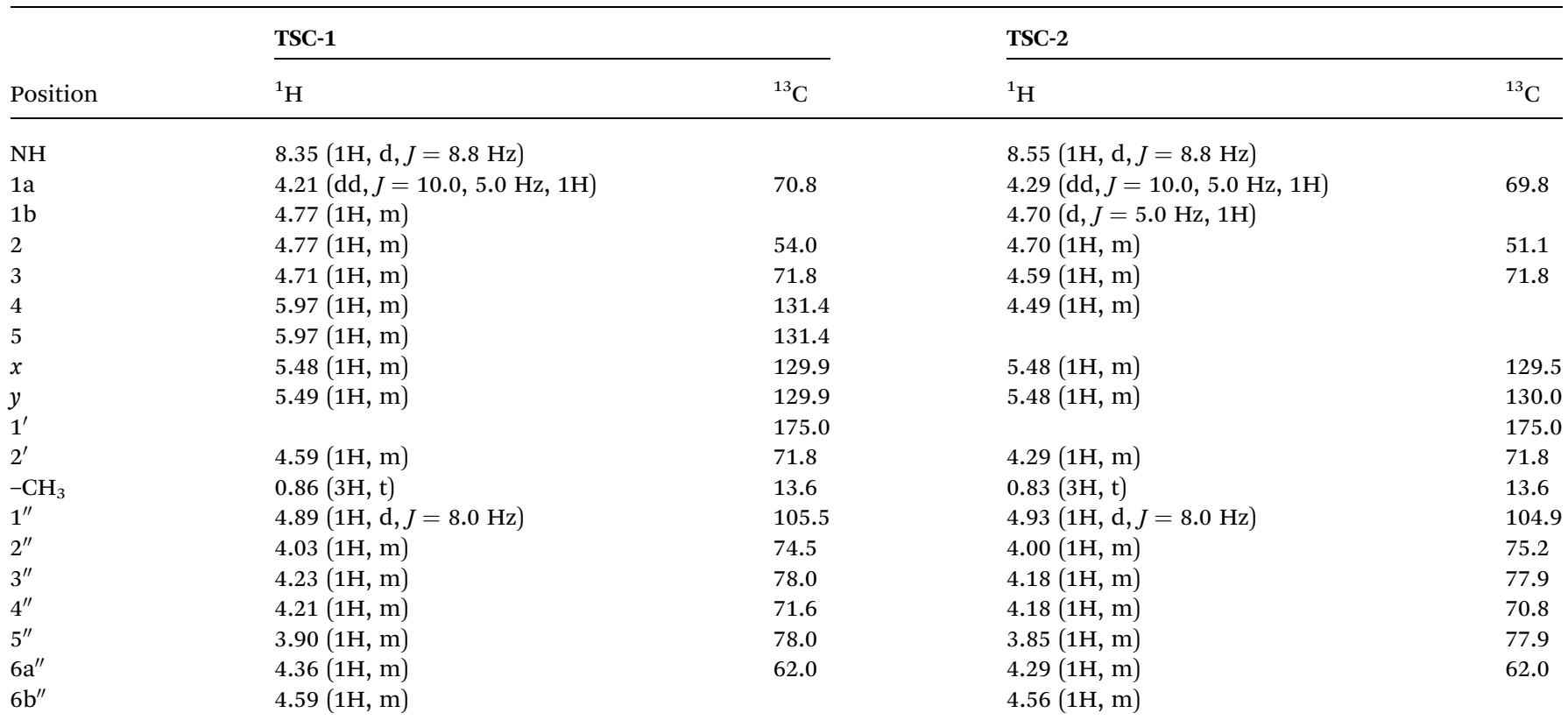

${ }^{a}$ Spectra were acquired at $23{ }^{\circ} \mathrm{C}$. Chemical shifts are given in $\delta(\mathrm{ppm})$ and are referenced to internal solvent signals for $\mathrm{C}_{5} \mathrm{D}_{5} \mathrm{~N}$ at $7.19\left(\delta_{\mathrm{H}}\right)$ and 123.5 $\left(\delta_{\mathrm{C}}\right) \mathrm{ppm} . X$ and $y$ are olefinic signals (double bond location).

\subsection{Spectral data}

2.4.1 $\beta$-Sitosteryl-3 $\beta$-D-glucopyranoside- $\boldsymbol{~}^{\prime}$ - $O$-methyl esters (TSS-1). ${ }^{1} \mathrm{H}$ NMR (chloroform- $\left.d, 300 \mathrm{MHz}, \mathrm{TMS}\right) \delta_{\mathrm{H}}(\mathrm{ppm}), 0.84$ $(3 \mathrm{H}$, terminal Me), $0.68(3 \mathrm{H}, \mathrm{s}, \mathrm{H}-18), 0.84(3 \mathrm{H}, \mathrm{s}, J=7.8 \mathrm{~Hz}, \mathrm{H}-$ 29), 0.87 (3H, s, H-26), 0.88 (3H, s, H-27), 1.01 (3H, s, H-19), 1.25 $\left(2 \mathrm{H}, \mathrm{s}, n \mathrm{CH}_{2}\right), 3.55(1 \mathrm{H}, \mathrm{m}, \mathrm{H}-3 \mathrm{a}), 5.36(1 \mathrm{H}, \mathrm{m}, \mathrm{H}-5)$ and $3.37-$ 4.53 (6H, m, H-2'-H-6'-glucose), $4.37\left(1 \mathrm{H}, \mathrm{d}, J=8.0 \mathrm{~Hz}, \mathrm{H}-1^{\prime}-\right.$ glucose). ${ }^{13} \mathrm{C}$ NMR (chloroform- $\left.d 100 \mathrm{MHz}, \mathrm{TMS}\right) \delta_{\mathrm{C}}(\mathrm{ppm})$, 37.24 (C-1), 29.70 (C-2), 79.54 (C-3), 38.88 (C-4), 140.26 (C-5), 122.19 (C-6), 31.92 (C-7), 31.92 (C-8), 50.14 (C-9), 36.71 (C-10), 21.04 (C-11), 39.74 (C-12), 42.31 (C-13), 56.73 (C-14), 24.28 (C15), 28.23 (C-16), 56.05 (C-17), 11.84 (C-18), 19.33 (C-19), 36.12 (C-20), 18.76 (C-21), 33.92 (C-22), 26.05 (C-23), 45.81 (C-24), 29.12 (C-25), 19.01 (C-26), 19.81 (C-27), 23.04 (C-28), 11.96 (C-

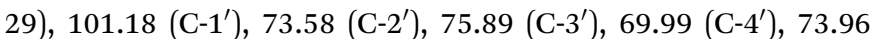
$\left(\mathrm{C}-5^{\prime}\right), 63.10\left(\mathrm{C}-6^{\prime}\right), 174.79\left(\mathrm{C}-1^{\prime \prime}\right), 34.21\left(\mathrm{C}-2^{\prime \prime}\right), 24.94\left(\mathrm{C}-3^{\prime \prime}\right)$, 22.57-29.76 $\left(n \mathrm{CH}_{2}\right)$ and 14.12 (terminal Me). ${ }^{19}$

2.4.2 $\beta$-Sitosteryl-3 $\beta$-D-glucopyranoside-6 ${ }^{\prime}$-O-palmitate (1). ${ }^{1} \mathrm{H}$ NMR (chloroform- $d$, $\left.300 \mathrm{MHz}, \mathrm{TMS}\right) \delta_{\mathrm{H}}(\mathrm{ppm}), 0.84(3 \mathrm{H}$, terminal Me), $0.68(3 \mathrm{H}, \mathrm{s}, \mathrm{H}-18), 0.84(3 \mathrm{H}, \mathrm{s}, J=7.8 \mathrm{~Hz}, \mathrm{H}-29)$, 0.87 (3H, s, H-26), 0.88 (3H, s, H-27), 1.01 (3H, s, H-19), 1.25 $\left(2 \mathrm{H}, \mathrm{s}, n \mathrm{CH}_{2}\right), 3.55(1 \mathrm{H}, \mathrm{m}, \mathrm{H}-3 \mathrm{a}), 5.36(1 \mathrm{H}, \mathrm{m}, \mathrm{H}-5)$ and 3.374.37 (6H, m, H-2'-H-6'-glucose), 4.37 (1H, d, $J=8.0 \mathrm{~Hz}, \mathrm{H}-1^{\prime}-$ glucose). ${ }^{13} \mathrm{C}$ NMR (chloroform- $\left.d, 100 \mathrm{MHz}, \mathrm{TMS}\right) \delta_{\mathrm{C}}(\mathrm{ppm})$, 37.24 (C-1), 29.70 (C-2), 79.54 (C-3), 38.88 (C-4), 140.26 (C-5), 122.19 (C-6), 31.92 (C-7), 31.92 (C-8), 50.14 (C-9), 36.71 (C-10), 21.04 (C-11), 39.74 (C-12), 42.31 (C-13), 56.73 (C-14), 24.28 (C15), 28.23 (C-16), 56.05 (C-17), 11.84 (C-18), 19.33 (C-19), 36.12 (C-20), 18.76 (C-21), 33.92 (C-22), 26.05 (C-23), 45.81 (C-24), 29.12 (C-25), 19.01 (C-26), 19.81 (C-27), 23.04 (C-28), 11.96 (C29), $101.18\left(\mathrm{C}-1^{\prime}\right), 73.58\left(\mathrm{C}-2^{\prime}\right), 75.89\left(\mathrm{C}-3^{\prime}\right), 69.99\left(\mathrm{C}-4^{\prime}\right), 73.96$
$\left(\mathrm{C}-5^{\prime}\right), 63.10\left(\mathrm{C}-6^{\prime}\right), 174.79\left(\mathrm{C}-1^{\prime \prime}\right), 34.21\left(\mathrm{C}-2^{\prime \prime}\right), 24.94\left(\mathrm{C}-3^{\prime \prime}\right), 29.30$ $\left.\left(\mathrm{C}-4^{\prime \prime}\right), 29.51\left(\mathrm{C}^{\prime \prime}\right)^{\prime \prime}\right), 29.71\left(\mathrm{C}-6^{\prime \prime}\right), 29.66\left(\mathrm{C}-7^{\prime \prime}-\mathrm{C}-12^{\prime \prime}\right), 29.36$ (C$\left.13^{\prime \prime}\right), 31.84\left(\mathrm{C}-14^{\prime \prime}\right), 22.68\left(\mathrm{C}-15^{\prime \prime}\right)$ and $14.12\left(\mathrm{C}-16^{\prime \prime}\right) .{ }^{19}$

2.4.3 $\beta$-Sitosterol (2). ${ }^{1} \mathrm{H}$ NMR (chloroform- $d$, $300 \mathrm{MHz}$, TMS) $\delta_{\mathrm{H}}(\mathrm{ppm}), 0.71(3 \mathrm{H}, \mathrm{s}, \mathrm{H}-19), 0.80(3 \mathrm{H}, \mathrm{s}, \mathrm{H}-26), 0.82(3 \mathrm{H}, \mathrm{s}$, $\mathrm{H}-27), 0.83$ (3H, s, H-29), 0.91, (3H, s, H-21), 1.03 (3H, s, H-18), $3.51(1 \mathrm{H}, \mathrm{m}, \mathrm{H}-3), 5.14(1 \mathrm{H}, \mathrm{m}, \mathrm{H}-23)$ and $5.31(1 \mathrm{H}, \mathrm{t}, \mathrm{H}-6) .{ }^{13} \mathrm{C}$ NMR (pyridine- $\left.d_{5}, 100 \mathrm{MHz}, \mathrm{TMS}\right) \delta_{\mathrm{C}}(\mathrm{ppm}), 37.4(\mathrm{C}-1), 30.2$ (C2), 108.5 (C-3), 42.3 (C-4), 140.9 (C-5), 121.9 (C-6), 32.0 (C-7), 32.1 (C-8), 50.3 (C-9), 36.9 (C-10), 21.2 (C-11), 39.9 (C-12), 42.5 (C-13), 56.8 (C-14), 24.5 (C-15), 28.5 (C-16), 56.2 (C-17), 11.9 (C-18), 19.4 (C-19), 36.3 (C-20), 21.2 (C-21), 34.2 (C-22), 26.4 (C-23), 51.3 (C24), 29.4 (C-25), 19.9 (C-26), 21.2 (C-27), 25.4 (C-28), and 12.1 (C-29). ${ }^{20}$

2.4.4 $\boldsymbol{\beta}$-Sitosterol-3 $\beta$-D-glucopyranoside (3). ${ }^{1} \mathrm{H}$ NMR (pyridine- $\left.d_{5}, 300 \mathrm{MHz}, \mathrm{TMS}\right) \delta_{\mathrm{H}}(\mathrm{ppm}), 0.71(3 \mathrm{H}, \mathrm{s}, \mathrm{H}-19), 0.80(3 \mathrm{H}, \mathrm{s}$, H-26), 0.82 (3H, s, H-27), 0.83 (3H, s, H-29), 0.91, (3H, s, H-21), 1.03 (3H, s, H-18), 3.55 (1H, m, H-3), 5.31 (1H, t, H-6) and 3.384.37 (5H, m, H-2'-H-5', glucose), $4.42\left(1 \mathrm{H}, \mathrm{d}, J=8.0 \mathrm{~Hz}, \mathrm{H}-1^{\prime}-\right.$ glucose). ${ }^{13} \mathrm{C}$ NMR (pyridine- $\left.d_{5}, 100 \mathrm{MHz}, \mathrm{TMS}\right) \delta_{\mathrm{C}}(\mathrm{ppm}), 37.4$ (C-1), 30.2 (C-2), 78.4 (C-3), 39.3 (C-4), 140.9 (C-5), 121.9 (C-6), 32.0 (C-7), 32.1 (C-8), 50.3 (C-9), 36.9 (C-10), 21.2 (C-11), 39.9 (C-12), 42.5 (C-13), 56.8 (C-14), 24.5 (C-15), 28.5 (C-16), 56.2 (C17), 11.9 (C-18), 19.4 (C-19), 36.3 (C-20), 19.0 (C-21), 34.2 (C22), 26.4 (C-23), 46.0 (C-24), 29.4 (C-25), 19.9 (C-26), 19.2 (C27), 23.4 (C-28), 12.1 (C-29), 102.6 (C-1'), 75.3 (C-2'), 78.1 (C$\left.3^{\prime}\right), 71.7\left(\mathrm{C}-4^{\prime}\right), 78.4\left(\mathrm{C}-5^{\prime}\right)$ and $62.8\left(\mathrm{C}-6^{\prime}\right) .{ }^{20}$

2.4.5 $\beta$-Stigmasterol (4). ${ }^{1} \mathrm{H}$ NMR (pyridine- $d_{5}, 300 \mathrm{MHz}$, TMS), $\delta_{\mathrm{H}}(\mathrm{ppm}), 0.66$ (3H, s, H-18), $0.86(3 \mathrm{H}, \mathrm{d}, J=7.7 \mathrm{~Hz}, \mathrm{H}-26)$, $0.84(3 \mathrm{H}, \mathrm{d}, J=6.1 \mathrm{~Hz}, \mathrm{H}-27), 0.88(3 \mathrm{H}, \mathrm{t}, J=6.1 \mathrm{~Hz}, \mathrm{H}-29), 1.01$ $(3 \mathrm{H}, \mathrm{s}, \mathrm{H}-19), 1.05(3 \mathrm{H}, \mathrm{d}, J=6.5 \mathrm{~Hz}, \mathrm{H}-21), 3.53$ (1H, m, H-3a), $5.15(\mathrm{dd}, J=8.4,15.1 \mathrm{~Hz}, \mathrm{H}-22), 5.02(\mathrm{dd}, J=8.4,15.1 \mathrm{~Hz}, \mathrm{H}-23)$ 
TSC-1

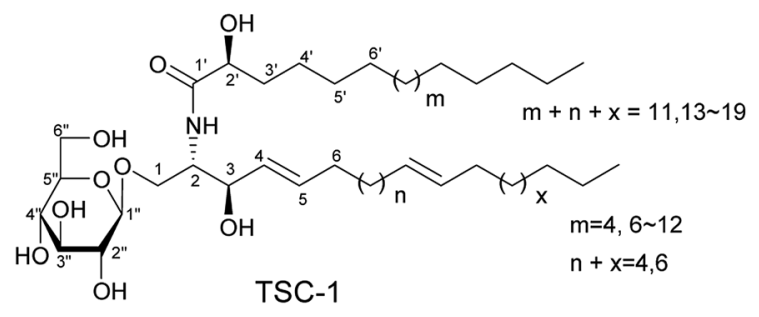

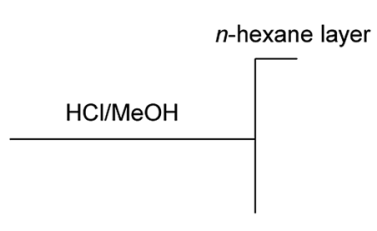

aqueous layer

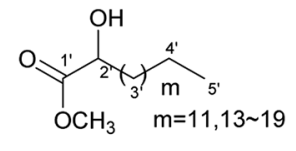

TSC-1-FAMEs

$+$

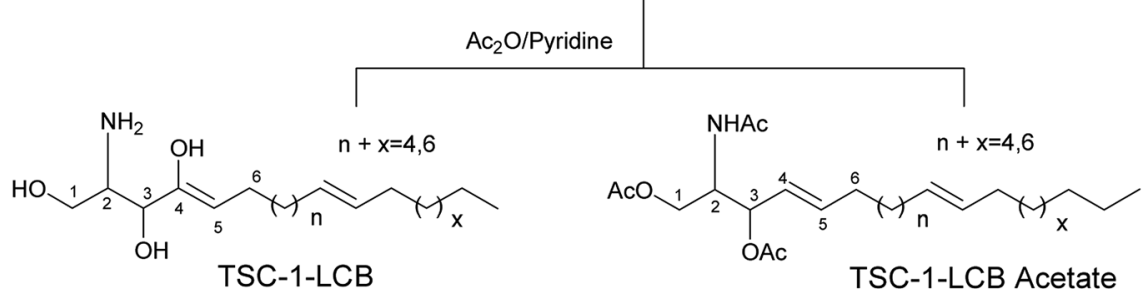

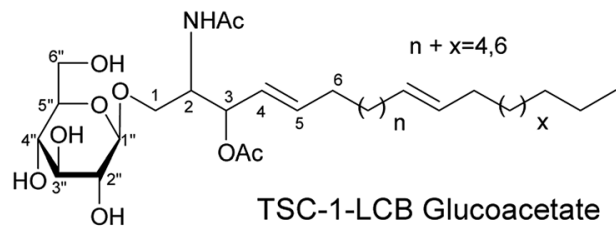

TSC-2

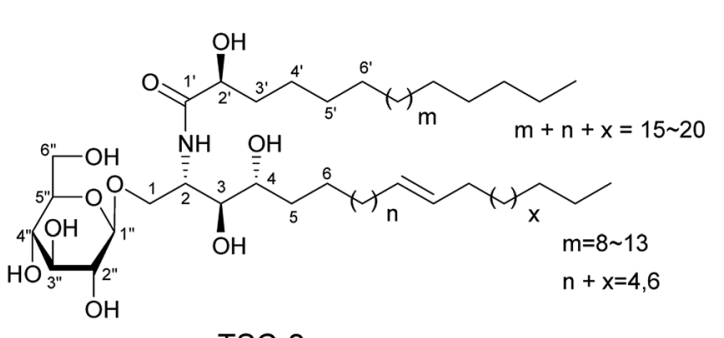

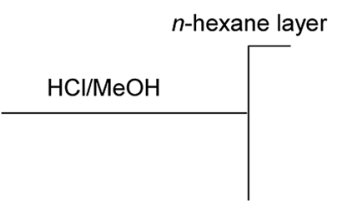

aqueous layer

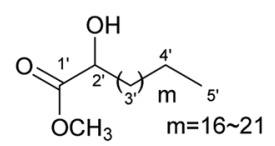

TSC-2-FAMES

$+$

TSC-2<smiles>CCCCC=CCCCC(O)C(O)C(N)CO</smiles>
$\mathrm{Ac}_{2} \mathrm{O} /$ Pyridine

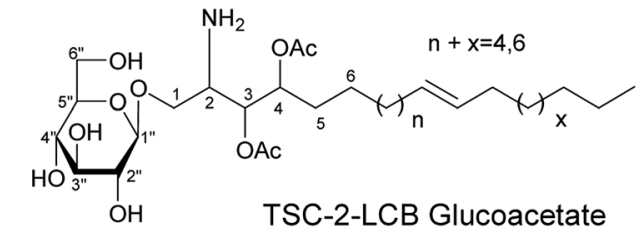

Fig. 1 Structures of TSC-1 and TSC-2.

and $5.33(1 \mathrm{H}, \mathrm{m}, \mathrm{H}-6) .{ }^{13} \mathrm{C}$ NMR (pyridine- $\left.d_{5}, 100 \mathrm{MHz}, \mathrm{TMS}\right) \delta_{\mathrm{C}}$ (ppm), 37.4 (C-1), 30.2 (C-2), 109.5 (C-3), 42.3 (C-4), 140.9 (C-5), 121.9 (C-6), 32.1 (C-7), 32.0 (C-8), 50.3 (C-9), 36.9 (C-10), 21.2 (C11), 39.8 (C-12), 42.3 (C-13), 56.9 (C-14), 24.5 (C-15), 28.3 (C-16), 56.9 (C-17), 12.1 (C-18), 19.4 (C-19), 40.7 (C-20), 21.4 (C-21),
138.8 (C-22), 129.4 (C-23), 51.4 (C-24), 32.1 (C-25), 21.2 (C-26), 19.1 (C-27), 25.7 (C-28) and 12.1 (C-29). ${ }^{19}$

2.4.6 $\beta$-Stigmasterol-3 $\beta$-D-glucopyranoside (5). ${ }^{1} \mathrm{H} \quad \mathrm{NMR}$ (pyridine- $\left.d_{5}, 300 \mathrm{MHz}, \mathrm{TMS}\right) \delta_{\mathrm{H}}(\mathrm{ppm}), 0.68(3 \mathrm{H}, \mathrm{s}, \mathrm{H}-18), 0.88$ $(3 \mathrm{H}, \mathrm{d}, J=6.4 \mathrm{~Hz}, \mathrm{H}-27), 0.89(3 \mathrm{H}, \mathrm{t}, J=7.5 \mathrm{~Hz}, \mathrm{H}-29), 0.92(3 \mathrm{H}$, 


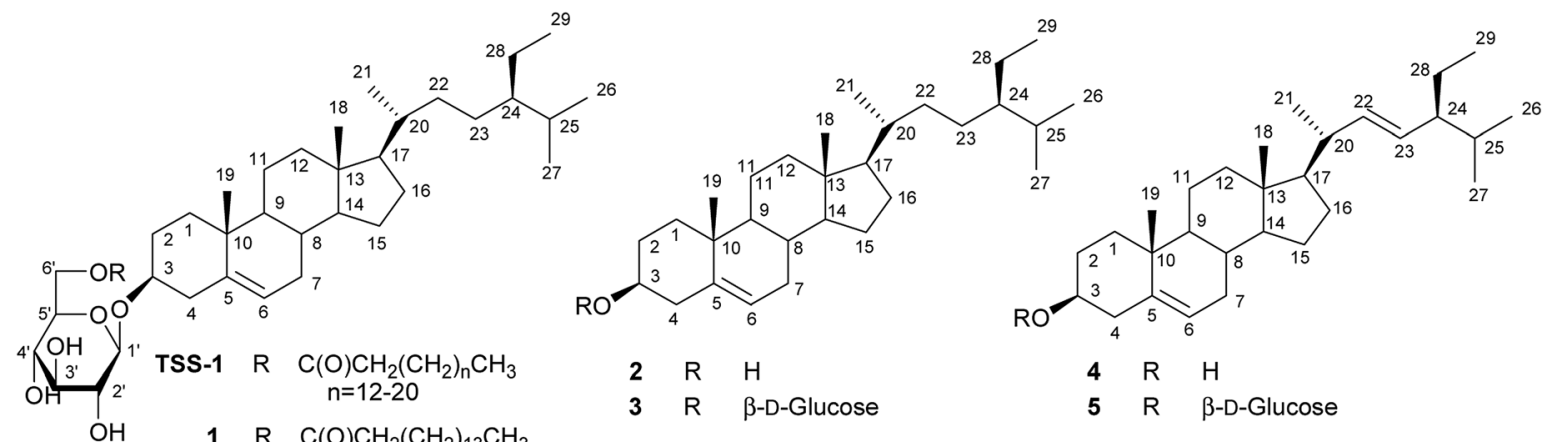

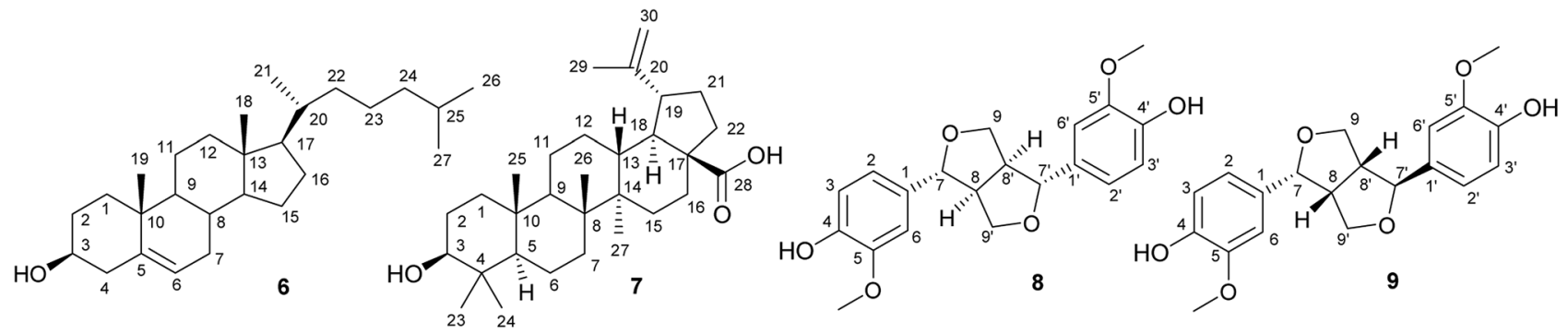<smiles>COc1cc([C@H]2c3cc(O)c(OC)cc3C[C@@](O)(CO)[C@@H]2CO)ccc1O</smiles><smiles>COc1cc(C[C@H](CO)[C@H](CO)Cc2ccc(O)c(OC)c2)ccc1O</smiles><smiles>COc1cc(C2c3cc(O)c(OC)cc3C[C@H](CO)[C@@H]2CO)ccc1O</smiles><smiles>O=c1cc(-c2ccc(O)c(O)c2)oc2cc(O)cc(O)c12</smiles>

Fig. 2 Structure of TSS-1 and comp. 1-13.

$\mathrm{d}, J=6.5 \mathrm{~Hz}, \mathrm{H}-26), 0.95(3 \mathrm{H}, \mathrm{s}, \mathrm{H}-19), 1.09(3 \mathrm{H}, \mathrm{d}, J=6.4 \mathrm{~Hz}, \mathrm{H}-$ 21), $4.317(1 \mathrm{H}, \mathrm{m}, \mathrm{H}-3 \mathrm{a}), 5.07$ (dd, $1 \mathrm{H}, J=8.9,15.1 \mathrm{~Hz}, \mathrm{H}-23)$, $5.23(\mathrm{dd}, 1 \mathrm{H}, J=8.7,15.1 \mathrm{~Hz}, \mathrm{H}-22), 5.33(\mathrm{H}-6)$ and 3.38-4.37 (5H, m, H-2'-H-5'-glucose), 4.42 (1H, d, $J=8.0 \mathrm{~Hz}, \mathrm{H}-1^{\prime}$-glucose). ${ }^{13} \mathrm{C}$ NMR (pyridine- $\left.d_{5}, 100 \mathrm{MHz}, \mathrm{TMS}\right) \delta_{\mathrm{C}}$ (ppm), 37.4 (C-1), 30.2 (C-2), 78.6 (C-3), 39.3 (C-4), 140.9 (C-5), 121.9 (C-6), 32.1 (C-7), 32.0 (C-8), 50.3 (C-9), 36.9 (C-10), 21.2 (C-11), 39.8 (C-12), 42.3 (C-13), 56.9 (C-14), 24.5 (C-15), 28.3 (C-16), 56.9 (C-17), 12.1 (C18), 19.4 (C-19), 40.7 (C-20), 21.4 (C-21), 138.8 (C-22), 129.4 (C23), 51.4 (C-24), 32.1 (C-25), 21.2 (C-26), 19.1 (C-27), 25.7 (C28), 12.5 (C-29), 102.5 (C-1'), 75.3 (C-2'), 78.1 (C-3'), 71.7 (C-4'), $78.4\left(\mathrm{C}-5^{\prime}\right)$ and $62.8\left(\mathrm{C}-6^{\prime}\right) .{ }^{20}$

2.4.7 Cholesterol (6). ${ }^{1} \mathrm{H}$ NMR (chloroform- $d$, $400 \mathrm{MHz}$, TMS) $\delta_{\mathrm{H}}(\mathrm{ppm}), 0.69,1.02$ (6H, s, C-18, C-19), 0.83 (6H, t, C-26, C27), $0.93(3 \mathrm{H}, \mathrm{d}, J=6.5 \mathrm{~Hz}, \mathrm{C}-21), 3.55(1 \mathrm{H}, \mathrm{m}, \mathrm{H}-3)$ and 5.38 $(1 \mathrm{H}, \mathrm{s}, \mathrm{H}-6) .{ }^{13} \mathrm{C}$ NMR (chloroform- $\left.d, 100 \mathrm{MHz}, \mathrm{TMS}\right) \delta_{\mathrm{C}}(\mathrm{ppm})$, 37.4 (C-1), 31.7 (C-2), 71.8 (C-3), 43.3 (C-4), 140.9 (C-5), 121.7 (C6), 32.0 (C-7), 31.7 (C-8), 50.3 (C-9), 36.9 (C-10), 21.2 (C-11), 39.9 (C-12), 36.2 (C-13), 56.8 (C-14), 24.5 (C-15), 28.5 (C-16), 56.2 (C17), 11.9 (C-18), 19.4 (C-19), 35.8 (C-20), 23.9 (C-21), 36.2 (C22), 23.9 (C-23), 39.6 (C-24), 22.6 (C-25) and 22.6 (C-26). ${ }^{21}$

2.4.8 Betulinic acid (7). ${ }^{1} \mathrm{H}$ NMR (chloroform- $d$, $400 \mathrm{MHz}$, TMS) $\delta_{\mathrm{H}}(\mathrm{ppm}), 4.93(1 \mathrm{H}$, brs, H-29b), 4.76 (1H, brs, H-29a), 3.52 (1H, m, H-18), 3.46 (1H, m, H-3), 1.78 (3H, s, H-30), 1.27, $(3 \mathrm{H}, \mathrm{s}$,
$\mathrm{H}-23), 1.21$ (3H, s, H-24), 0.84 (3H, s, H-25), 1.04 (3H, s, H-26), $1.05(3 \mathrm{H}, \mathrm{s}, \mathrm{H}-27) .{ }^{13} \mathrm{C}$ NMR (pyridine- $\left.d_{5}, 100 \mathrm{MHz}, \mathrm{TMS}\right) \delta_{\mathrm{C}}$ (ppm), 39.22 (C-1), 28.24 (C-2), 78.07 (C-3), 39.48 (C-4), 55.85 (C5), 18.73 (C-6), 34.76 (C-7), 41.05 (C-8), 50.89 (C-9), 37.55 (C-10), 21.14 (C-11), 26.05 (C-12), 38.55 (C-13), 42.79 (C-14), 30.23 (C15), 32.83 (C-16), 56.58 (C-17), 47.73 (C-18), 49.69 (C-19), 151.29 (C-20), 29.96 (C-21), 37.46 (C-22), 28.62 (C-23), 16.32 (C24), 16.32 (C-25), 19.42 (C-26), 14.85 (C-27), 178.86 (C-28), $109.93(\mathrm{C}-29)$ and $19.42(\mathrm{C}-30){ }^{22}$

2.4.9 (+)-Epipinoresinol (8). ${ }^{1} \mathrm{H}$ NMR (chloroform- $d$, 400 MHz, TMS) $\delta_{\mathrm{H}}(\mathrm{ppm}), 6.97-6.76\left(6 \mathrm{H}, \mathrm{m}, \mathrm{H}-6^{\prime}, 6^{\prime \prime}, 3^{\prime}, 3^{\prime \prime}, 2^{\prime}, 2^{\prime \prime}\right)$, 3.95 (3H, s, H-5', OMe), 3.85 (3H, s, H-5", OMe), 3.83-3.75 (2H, $\left.\mathrm{m}, \mathrm{H}-9,9^{\prime}\right)$, and $3.37-3.28\left(2 \mathrm{H}, \mathrm{m}, \mathrm{H}-8,8^{\prime}\right) .{ }^{13} \mathrm{C}$ NMR (chloroform- $d 100 \mathrm{MHz}$, TMS) $\delta_{\mathrm{C}}(\mathrm{ppm}), 146.69,146.39$ (C-3, 3'), 145.29, 144.56 (C-4, 4'), 133.00, 130.30 (C-1, 1'), 119.17, 116.37 (C-6, 6'), 114.19 (C-5, 5'), 108.47, 108.30 (C-2, 2'), 87.71, 82.07 (C-7, 7'), 70.95, 69.67 (C-9, 9') 55.97, 55.91 (3, 3' -OMe) and 54.46, 50.1 $\left(\mathrm{C}-8,8^{\prime}\right) .{ }^{23}$

2.4.10 (+)-Pinoresinol (9). ${ }^{1} \mathrm{H}$ NMR (chloroform- $d, 400$ MHz, TMS) $\delta_{\mathrm{H}}(\mathrm{ppm}), 6.9\left(2 \mathrm{H}, \mathrm{d}, J=1.5 \mathrm{~Hz}, \mathrm{H}-6^{\prime}, 6^{\prime \prime}\right), 6.78(2 \mathrm{H}$, $\left.\mathrm{d}, J=8.0 \mathrm{~Hz}, \mathrm{H}-3^{\prime}, 3^{\prime \prime}\right), 6.71\left(2 \mathrm{H}, \mathrm{dd}, J=1.5,8.0 \mathrm{~Hz}, \mathrm{H}-2^{\prime}, 2^{\prime \prime}\right)$, $4.73\left(2 \mathrm{H}, \mathrm{d}, J=5.0 \mathrm{~Hz}, \mathrm{H}-7,7^{\prime}\right), 4.24\left(2 \mathrm{H}, \mathrm{dd}, J=7.0,9.5 \mathrm{~Hz}, \mathrm{H}^{-}\right.$ 9), $3.88\left(2 \mathrm{H}, \mathrm{dd}, J=4.0,9.5 \mathrm{~Hz}, \mathrm{H}-9^{\prime}\right), 3.80$ (6H, s, H-5', $\left.5^{\prime \prime}, \mathrm{OMe}\right)$ and $3.09\left(2 \mathrm{H}, \mathrm{m}, \mathrm{H}-8,8^{\prime}\right) .{ }^{13} \mathrm{C}$ NMR (chloroform- $d 100 \mathrm{MHz}$, 
TMS) $\delta_{\mathrm{C}}(\mathrm{ppm}), 146.67\left(\mathrm{C}-3,3^{\prime}\right), 145.19\left(\mathrm{C}-4,4^{\prime}\right), 132.85\left(\mathrm{C}-1,1^{\prime}\right)$, $118.93\left(\mathrm{C}-6,6^{\prime}\right), 114.23\left(\mathrm{C}-5,5^{\prime}\right), 108.55\left(\mathrm{C}-2,2^{\prime}\right), 85.84\left(\mathrm{C}-7,7^{\prime}\right)$, $71.63\left(\mathrm{C}-9,9^{\prime}\right), 55.92\left(3,3^{\prime}-\mathrm{OMe}\right)$ and $54.12\left(\mathrm{C}-8,8^{\prime}\right) .{ }^{23}$

2.4.11 (+)-Cycloolivil (10). ${ }^{1} \mathrm{H}$ NMR (methanol- $d_{4}, 400 \mathrm{MHz}$, TMS) $\delta_{\mathrm{H}}(\mathrm{ppm}), 6.75(1 \mathrm{H}, \mathrm{d}, J=8.0 \mathrm{~Hz}, \mathrm{H}-5), 6.73(1 \mathrm{H}, \mathrm{d}, J=$ $8.0 \mathrm{~Hz}, \mathrm{H}-2), 6.67$ (1H, dd, $J=8.0,2.0 \mathrm{~Hz}, \mathrm{H}-6), 6.61\left(1 \mathrm{H}, \mathrm{s}, \mathrm{H}-2^{\prime}\right)$, $6.12\left(1 \mathrm{H}, \mathrm{s}, \mathrm{H}-5^{\prime}\right), 4.62(1 \mathrm{H}, \mathrm{m}, \mathrm{H}-7), 3.82$ (1H, m, H-9b), 3.80 (3H, s, OMe), 3.79 (1H, d, J=11.2 Hz, H-9'b), 3.75 (3H, s, OMe), 3.59 (2H, m, H-9'a), H-9b, 3.37 (1H, m, H-7'b), 2.61 (1H, m, $\left.7^{\prime}\right)$ and $2.05(1 \mathrm{H}, \mathrm{m}, \mathrm{H}-8) .{ }^{13} \mathrm{C}$ NMR (methanol- $d_{4}, 100 \mathrm{MHz}, \mathrm{TMS}$ ) $\delta_{\mathrm{C}}(\mathrm{ppm}), 149.23,147.39\left(\mathrm{C}-3,3^{\prime}\right)$ 146.20, $145.39\left(\mathrm{C}-4,4^{\prime}\right), 138.02$ (C-1'), 133.75 (C-1), 126.60 (C-6'), 123.12 (C-6), 117.38, 116.01, 113.22, $112.35\left(\mathrm{C}-5,5,2,2^{\prime}\right), 74.90\left(\mathrm{C}-8^{\prime}\right), 69.50(\mathrm{C}-9), 61.00\left(\mathrm{C}-9^{\prime}\right)$, 56.35, 56.33 (OMe), 47.90 (C-8), $45.0(\mathrm{C}-7)$ and $40.10\left(\mathrm{C}-7^{\prime}\right) .{ }^{24}$

2.4.12 (+)-Secolariciresinol (11). ${ }^{1} \mathrm{H}$ NMR (methanol- $d_{4}, 400$ MHz, TMS $) \delta_{\mathrm{H}}(\mathrm{ppm}), 6.66\left(2 \mathrm{H}, \mathrm{d}, J=8.0 \mathrm{~Hz}, \mathrm{H} 5, \mathrm{H}-5^{\prime}\right), 6.64(2 \mathrm{H}$, $\left.\mathrm{d}, J=2.0 \mathrm{~Hz}, \mathrm{H}-2,2^{\prime}\right), 6.53\left(2 \mathrm{H}, \mathrm{dd}, J=8.0,2.0 \mathrm{~Hz}, \mathrm{H}-6,6^{\prime}\right), 3.73$ $\left(6 \mathrm{H}, \mathrm{s}, \mathrm{H}-3,3^{\prime},-\mathrm{OCH}_{3}\right), 3.57$ (4H, m, H-9, 9'), 2.68 (2H, m, H-7'a, 7a) and $1.90\left(2 \mathrm{H}\right.$, br m, H-8, $\left.8^{\prime}\right) .{ }^{13} \mathrm{C}$ NMR (methanol- $d_{4}, 100$ MHz, TMS) $\delta_{\mathrm{C}}(\mathrm{ppm}), 79.9$ (C-2), 43.5 (C-3), 197.2 (C-4), 165.2 (C5), 96.7 (C-6), 167.3 (C-7), 95.8 (C-8), 164.3 (C-9), 103.2 (C-10), $131.5\left(\mathrm{C}-1^{\prime}\right), 114.7\left(\mathrm{C}-2^{\prime}\right), 146.1\left(\mathrm{C}-3^{\prime}\right), 147.1\left(\mathrm{C}-4^{\prime}\right), 116.0\left(\mathrm{C}-5^{\prime}\right)$ and $119.2\left(\mathrm{C}-6^{\prime}\right){ }^{25}$

2.4.13 (+)-Isolariciresinol (12). ${ }^{1} \mathrm{H}$ NMR (methanol- $d_{4}, 400$ MHz, TMS) $\delta_{\mathrm{H}}(\mathrm{ppm}), 6.75\left(2 \mathrm{H}, \mathrm{d}, J=8.0 \mathrm{~Hz}, \mathrm{H}-5,5^{\prime}\right), 6.67(2 \mathrm{H}$, $\left.\mathrm{d}, J=2.0 \mathrm{~Hz}, \mathrm{H}-2,2^{\prime}\right), 6.43\left(2 \mathrm{H}, \mathrm{m}, \mathrm{H}-6,6^{\prime}\right), 6.2\left(2 \mathrm{H}, \mathrm{s}, \mathrm{H}-6,6^{\prime}\right)$, 3.80, 3.82 (6H, s, H-3, $\left.3^{\prime}-\mathrm{OMe}\right), 3.74-3.34$ (4H, m, H-9, $\left.9^{\prime}\right), 2.77$ $\left(2 \mathrm{H}, \mathrm{m}, \mathrm{H}-7^{\prime}, 7\right)$ and $1.99\left(2 \mathrm{H}, \mathrm{m}, \mathrm{H}-8,8^{\prime}\right) .{ }^{13} \mathrm{C}$ NMR (methanol$\left.d_{4}, 100 \mathrm{MHz}, \mathrm{TMS}\right) \delta_{\mathrm{C}}(\mathrm{ppm}), 138.6$ (C-1), 113.8 (C-2), 149.0 (C-3), 145.9 (C-4), 117.4 (C-5), 129.0 (C-6), 39.9 (C-7), 48.0 (C-8), 65.9 (C9), $134.2\left(\mathrm{C}-1^{\prime}\right), 112.4\left(\mathrm{C}-2^{\prime}\right), 147.2\left(\mathrm{C}-3^{\prime}\right), 145.3\left(\mathrm{C}-4^{\prime}\right), 116.0(\mathrm{C}-$ $\left.5^{\prime}\right), 129.0\left(\mathrm{C}-6^{\prime}\right), 39.9\left({\mathrm{C}-7^{\prime}}^{\prime}\right), 48.0\left(\mathrm{C}-8^{\prime}\right), 62.2\left(\mathrm{C}-9^{\prime}\right)$ and 56.4 $(\mathrm{OMe}){ }^{23}$

2.4.14 (+)-Eriodictyol (13). ${ }^{1} \mathrm{H}$ NMR (methanol- $d_{4}, 400 \mathrm{MHz}$, TMS) $\delta_{\mathrm{H}}(\mathrm{ppm}), 6.91\left(1 \mathrm{H}, \mathrm{s}, \mathrm{H}-2^{\prime}\right), 6.78\left(1 \mathrm{H}, \mathrm{s}, \mathrm{H}-5^{\prime}\right), 5.90(1 \mathrm{H}, \mathrm{d}, J$ $=2.2, \mathrm{H}-8), 5.88(1 \mathrm{H}, \mathrm{d}, J=2.2, \mathrm{H}-6), 5.28(1 \mathrm{H}, \mathrm{dd}, J=12.8$; $3.0 \mathrm{~Hz}, \mathrm{H}-2), 3.06(1 \mathrm{H}, \mathrm{dd}, J=17.2,12.8 \mathrm{~Hz}, \mathrm{H}-3 \mathrm{a})$ and $2.66(1 \mathrm{H}$, $\mathrm{dd}, J=17.2,3.0 \mathrm{~Hz}, \mathrm{H}-3 \mathrm{~b}) .{ }^{13} \mathrm{C}$ NMR (methanol- $d_{4}, 100 \mathrm{MHz}$, TMS) $\delta_{\mathrm{C}}(\mathrm{ppm}), 80.53$ (C-2), 44.13 (C-3), 197.80 (C-4), 165.5 (C-5), 97.07 (C-6), 168.50 (C-7), 96.20 (C-8), 164.87 (C-9), 103.35 (C-10), $131.80\left(\mathrm{C}-1^{\prime}\right), 114.73\left(\mathrm{C}-2^{\prime}\right), 146.54\left(\mathrm{C}-3^{\prime}\right), 146.92\left(\mathrm{C}-4^{\prime}\right), 116.28(\mathrm{C}-$ $\left.5^{\prime}\right)$ and $119.29\left(\mathrm{C}-6^{\prime}\right){ }^{26}$

\section{Results and discussion}

Chromatographic separation of the $n$-hexane fraction, from the chloroform/methanol crude extract of $T$. sanguinea whole plant lead to the isolation of two glucocerebroside molecular species TSC-1 and TSC-2, and one $\beta$-sitosteryl-3 $\beta$-D-glucopyranoside- 6 - $-O$ fatty acid ester molecular species TSS-1, together with seven known triterpenes: $\beta$-sitosteryl-3 $\beta$-D-glucopyranoside- 6 '-O-palmitate $1, \beta$-sitosterol 2 , $\beta$-sitosterol-3 $\beta$-D-glucopyranoside 3 , $\beta$-stigmasterol 4, $\beta$-stigmasterol-3 $\beta$-D-glucopyranoside 5 , cholesterol 6 and betulinic acid 7. Five known lignans: (+)-epipinoresinol 8, (+)-pinoresinol 9, (+)-cycloolivil, 10, (+)-secoisolariciresinol 11 and $(+)$-isolariciresinol 12 and one known flavanone (+)-eriodictyol 13, were also isolated from the ethyl acetate fraction of $T$. sanguinea whole plant. The known compounds were identified by comparison with authentic samples or reported spectral and physical data. See Fig. 1 and 2; Spectral data.

\subsection{Chemistry}

3.1.1 $\beta$-sitosteryl-3 $\beta$-D-glucopyranoside- $6^{\prime}-O$-fatty acid methyl ester molecular species (TSS-1). TSS-1 (94 mg) was obtained as a white amorphous solid, and showed as a single spot on silica gel TLC plate. It exhibited a strong absorption at $3350 \mathrm{~cm}^{-1}$ indicating the presence of a hydroxyl group and a band at $1720 \mathrm{~cm}^{-1}$ (carbonyl) indicating a stretching of a normal aliphatic ester. The ${ }^{1} \mathrm{H}$ and ${ }^{13} \mathrm{C}$ NMR spectra reveal characteristic signals for a $\beta$ sitosterol glucoside and long fatty acid methyl esters. A series of molecular ion peaks in the positive FAB-MS spectra confirmed the possible structure of TSS-1. See Fig. 2 and Spectral data.

The NMR spectral data of TSS-1 in $\mathrm{CDCl}_{3}$ showed resonances of a carboxylic acid group $\left(\delta_{\mathrm{C}} 174.8\right)$, a long methylene chain centered at $\delta_{\mathrm{H}} 1.25,\left(\delta_{\mathrm{C}} 29.1-29.9\right)$ and overlapped methyls at $\delta_{\mathrm{H}}$ $0.68-0.85\left(\delta_{\mathrm{C}} 14.1\right)$, indicating normal type terminal methyls of the fatty acids. The characteristic signals of a sitosterol skeleton were determined as follows: a methine proton at $\delta_{\mathrm{H}} 3.55(1 \mathrm{H}, \mathrm{m}$, $\left.\mathrm{H}-3, \delta_{\mathrm{C}} 79.5\right)$ and an olefinic proton signal at $\delta_{\mathrm{H}} 5.38(1 \mathrm{H}, \mathrm{m}, \mathrm{H}-$ $\left.5, \delta_{\mathrm{C}} 140.3\right)$ were assigned as $\mathrm{C}-3$ and $\mathrm{C}-5$ respectively. Two angular methyl protons at $\delta_{\mathrm{H}} 0.68(3 \mathrm{H}, \mathrm{s})$ and $1.01(3 \mathrm{H}, \mathrm{s})$, corresponding to $\delta_{\mathrm{C}} 11.8$ and 19.3 were assigned as C-18 and C19 respectively. The proton signals at $\delta_{\mathrm{H}} 0.87\left(3 \mathrm{H}, \mathrm{s}, \delta_{\mathrm{C}} 19.0\right.$; C$26)$ and $0.88\left(3 \mathrm{H}, \mathrm{s}, \delta_{\mathrm{C}} 19.8 ; \mathrm{C}-27\right)$ indicated the presence of an isopropenyl group in the molecular structure. The proton signal at $\delta_{\mathrm{H}} 0.84\left(3 \mathrm{H}, \mathrm{t}, J=7.8 \mathrm{~Hz} ; \delta_{\mathrm{C}} 11.9\right)$ was assigned as C-29. All other NMR assignments were in agreement with known $\beta$ sitosteryl-3 $\beta$-D-glucopyranoside-6'-O-fatty acid methyl esters. ${ }^{19}$ Characteristic signals indicative of a presence of a monosaccharide moiety at $\delta_{\mathrm{H}} 3.38-4.53,6 \mathrm{H}$ with an anomeric proton signal at $\delta_{\mathrm{H}} 4.37\left(1 \mathrm{H}, \mathrm{d}, J=7.8 \mathrm{~Hz} ; \delta_{\mathrm{C}} 101.2\right)$ were observed. See Fig. 1 and Spectral data.

To identify the sugar moiety in TSS-1, the methylated sugar moiety in the aqueous layer after methanolysis was analysed by HPLC against standard sugars (glucose and galactose) and identified as glucose (glucose $t_{\mathrm{R}}=14.11 \mathrm{~min}$, galactose $t_{\mathrm{R}}=$ $13.27 \mathrm{~min}$ ). The coupling constant of the anomeric proton at $\delta_{\mathrm{H}}$ $4.38(1 \mathrm{H}, \mathrm{d}, J=8.0 \mathrm{~Hz})$ and the chemical shift of the anomeric carbon $\delta_{\mathrm{C}}(101.2)$ confirmed the $\beta$-configuration of the glucopyranoside moiety ( $\alpha$-glucopyranoside: $\left.J=3.7 \mathrm{~Hz} ; \delta_{\mathrm{C}} 98.5\right) .{ }^{18}$ The absolute configuration of the sugar moiety was determined by the Tanaka et al. method. ${ }^{18}$ Direct HPLC analysis of the reaction mixture of the sugar moiety exhibited a peak at $t_{\mathrm{R}}=$ $18.7 \mathrm{~min}$, which were coincided with the aryl-isothiocyanate derivative of $\mathrm{D}$-glucose, confirming the absolute configuration of the sugar moiety (L-glucose $\left.t_{\mathrm{R}}=19.22 \mathrm{~min}\right) .{ }^{18}$

The positive FAB-MS spectral data showed a series of molecular ion peaks at $m / z: 851,865,879,893,907,921,935$, 949, 963 and $977[\mathrm{M}+\mathrm{Na}]^{+}$. Therefore, TSS-1 is presumed to be a molecular species consisting of $\beta$-sitosteryl-3 $\beta$-D-glucopyranoside- $6^{\prime}-O$-fatty acid methyl ester possessing mainly a hydroxy fatty acid moiety (normal type terminal methyl groups at $\delta_{\mathrm{C}}$ 14.2) and a $\beta$-D-glucopyranose moiety. The core structure of the 
$\beta$-sitosterol-3 $\beta$-D-glucopyranose skeleton of TSS-1 was characterized by comparison of its ${ }^{13} \mathrm{C}$ NMR spectral data with that of known $\beta$-sitosterol-3 $\beta$-D-glucopyranose-6-O-fatty acid esters. ${ }^{19}$ See Fig. 2 and Spectral data.

3.1.2 Cerebrosides from TSC-1. TSC-1 (30 mg) was obtained as a white amorphous solid, and showed as a single spot on silica gel TLC plate. Strong hydroxy $\left(3422 \mathrm{~cm}^{-1}\right)$ and amide absorptions $\left(1650,1540 \mathrm{~cm}^{-1}\right)$ were observed in the IR spectrum.

The NMR spectral data of TSC-1 in $\mathrm{C}_{5} \mathrm{D}_{5} \mathrm{~N}$ showed resonances of a secondary amide proton doublet at $\delta_{\mathrm{H}} 8.35(1 \mathrm{H}, \mathrm{d}, J$ $=8.8 \mathrm{~Hz})$, a long methylene chain, centered at $\delta_{\mathrm{H}} 1.26,\left(\delta_{\mathrm{C}}\right.$ 29.1-29.3) and overlapped methyls at $\delta_{\mathrm{H}} 0.86\left(\delta_{\mathrm{C}} 13.6\right)$, indicating the presence of a sphingolipid skeleton. Characteristic signals indicative of a monosaccharide moiety at $\delta_{\mathrm{H}} 3.90-4.89$ $(6 \mathrm{H})$, with the anomeric proton signal at $\delta_{\mathrm{H}} 4.89(1 \mathrm{H}, \mathrm{d}, J=8.0$ $\left.\mathrm{Hz} ; \delta_{\mathrm{C}} 105.5\right)$ were observed. The characteristic resonances for the 2-amino-1,3,2'-triol region of the hydrocarbon chain were observed at $\delta_{\mathrm{H}} 4.77(1 \mathrm{H}, \mathrm{m}, \mathrm{H}-2), 4.59\left(1 \mathrm{H}, \mathrm{m}, \mathrm{H}-2^{\prime}\right), 4.77(1 \mathrm{H}$, $\mathrm{m}, \mathrm{H}-1 \mathrm{~b}), 4.21(1 \mathrm{H}, \mathrm{m}, \mathrm{H}-1 \mathrm{a}), 4.71(1 \mathrm{H}, \mathrm{m}, \mathrm{H}-3)$ corresponding to the following ${ }^{13} \mathrm{C}$ NMR data: $\delta_{\mathrm{C}} 54.0(\mathrm{C}-2), 71.8\left(\mathrm{C}-2^{\prime}\right), 70.8$ (C-1), 71.8 (C-3), and an amide carbonyl signal at $\delta_{\mathrm{C}} 175.0$ (C$\left.1^{\prime}\right)$. See Fig. 1 and Table 1.

The presence of two disubstituted double bonds at $\delta_{\mathrm{C}} 131.4$ (2CH, C-4, C-5) and $129.9(2 \mathrm{CH})$ were observed. The $E$ geometry for the double bonds was supported from the characteristic chemical shift of the allylic carbons at $\delta_{\mathrm{C}} 32.2,32.3$ and 34 . ( $Z$ geometry $=\delta_{\mathrm{C}} 27.0$ ).

The positive FAB-MS spectral data showed a series of molecular ion peaks due to $[\mathrm{M}+\mathrm{Na}]^{+}$at $m / z: 736,764,778,792$, $806,820,834$ and 848 . TSC-1 is hence presumed to be a molecular species consisting of a sphingosine-type cerebroside possessing mainly a 2-hydroxy fatty acid moiety (normal type terminal methyls at $\left.\delta_{\mathrm{C}} 13.6\right)$ and a $\beta$-D-glucopyranose moiety.

The sphingosine skeleton was characterized by comparison of its ${ }^{1} \mathrm{H}$ and ${ }^{13} \mathrm{C}$ NMR spectral data (Fig. 1 and Table 1 ) with that of known cerebrosides. ${ }^{27}$ The relative stereochemistry of the ceramide moiety is presumed to be $\left(2 S, 3 R, 4 E, 2^{\prime} R\right)$ since the characteristic ${ }^{13} \mathrm{C}$ NMR signals $\left(\mathrm{C}-1,2,3,4,1^{\prime}\right.$ and $\left.2^{\prime}\right)$ in addition to the optical rotation value of $\left([\alpha]_{\mathrm{D}}^{20}=-31.3\right)$ are in good agreement with those of the sphingosine-type glucocerebroside molecular species possessing a $2 S, 3 R, 4 E, 2^{\prime} R$ configuration. ${ }^{27}$ The analysis of the methylated sugar against standard sugars (glucose and galactose) using HPLC indicated that the sugar moiety in TSC-1 was glucose (glucose $t_{\mathrm{R}}=$ $14.11 \mathrm{~min}$, galactose $t_{\mathrm{R}}=13.27 \mathrm{~min}$ ). The coupling constant of the anomeric proton at $\delta_{\mathrm{H}} 4.89(1 \mathrm{H}, \mathrm{d}, J=8.0 \mathrm{~Hz})$ and the chemical shift of the anomeric carbon $\delta_{\mathrm{C}}(105.5)$ confirmed the $\beta$-configuration of the glucopyranoside moiety ( $\alpha$-glucopyranoside: $\left.J=3.7 \mathrm{~Hz} ; \delta_{\mathrm{C}} 98.5\right) .{ }^{18}$ The absolute configuration of the sugar moiety was determined using the Tanaka et al. method. ${ }^{18}$ HPLC analysis of the reaction mixture exhibited a peak at $t_{\mathrm{R}}=18.68 \mathrm{~min}$, which were coincided with the arylisothiocyanate derivative of $\mathrm{D}_{\text {-glucose, }}$ confirming the absolute configuration of the sugar moiety (L-glucose $t_{\mathrm{R}}=19.22 \mathrm{~min}$ ).

To determine the length of the FAMEs and LCB in the glucocerebrosides, the methanolysis products of TSC-1 were subjected to ${ }^{1} \mathrm{H},{ }^{13} \mathrm{C}$ NMR, and FAB-MS analyses. Molecular ion peaks at 286, 314, 327, 343, 357, 371, 385, 399 and 413 [M + $\mathrm{H}]^{+}$indicated the presence of $\mathrm{C}-16, \mathrm{C}-18-\mathrm{C}-25$ fatty acid methyl esters, possessing normal terminal methyl groups $\left(\delta_{\mathrm{C}} 13.6\right)$. The LCB mixture showed molecular ion peaks at $395[\mathrm{M}+]$ (LCB) and $424[\mathrm{M}+\mathrm{H}]^{+}$(LCB acetate) indicating the presence of a C-16 LCB, while $652[\mathrm{M}+\mathrm{Na}]^{+}$(LCB glucoacetate) indicated the presence of a C-18 LCB in TSC-1. See Fig. 1.

3.1.3 Cerebrosides from TSC-2. TSC-2 ( $45 \mathrm{mg}$ ) was obtained as a white amorphous solid, seen as a single spot on normalphase (silica gel) TLC plate. It exhibited strong hydroxy $\left(3289 \mathrm{~cm}^{-1}\right)$ and amide absorptions $\left(1650,1540 \mathrm{~cm}^{-1}\right)$ in the IR spectrum.

The NMR spectra of TSC-2 in $\mathrm{C}_{5} \mathrm{D}_{5} \mathrm{~N}$ showed resonances for a secondary amide proton doublet at $\delta_{\mathrm{H}} 8.55(1 \mathrm{H}, \mathrm{d}, J=8.0 \mathrm{~Hz})$, protons of a long methylene chain, centered at $\delta_{\mathrm{H}} 1.25,\left(\delta_{\mathrm{C}} 29.1-\right.$ $29.8)$ and overlapped methyls at $\delta_{\mathrm{H}} 0.83\left(\delta_{\mathrm{C}} 13.6\right)$, indicating the presence of a sphingolipid skeleton. The proton signals at $\delta_{\mathrm{H}}$ $3.85-4.93(6 \mathrm{H})$ and the anomeric proton signal at $\delta_{\mathrm{H}} 4.93(1 \mathrm{H}, \mathrm{d}$, $\left.J=8.0 \mathrm{~Hz} ; \delta_{\mathrm{C}} 104.9\right)$ confirmed the presence of a monosaccharide moiety.

TSC-2 showed characteristic resonances for the 2-amino$1,3,4,2^{\prime}$-tiol region of hydrocarbon chain with proton signals at $\delta_{\mathrm{H}}$ 4.70 (1H, m, H-2), 4.29 (1H, m, H-2'), 4.70 (1H, m, H-1b), $4.29(1 \mathrm{H}$, $\mathrm{m}, \mathrm{H}-1 \mathrm{a}), 4.59(1 \mathrm{H}, \mathrm{m}, \mathrm{H}-3)$, and $4.49(1 \mathrm{H}, \mathrm{m}, \mathrm{H}-4)$, corresponding to the following ${ }^{13} \mathrm{C}$ NMR data: $\delta_{\mathrm{C}} 51.1(\mathrm{C}-2), 71.8\left(\mathrm{C}-2^{\prime}\right), 69.8$ (C1), 71.8 (C-3) and an amide carbonyl signal at $\delta_{\mathrm{C}} 175.0\left(\mathrm{C}-1^{\prime}\right)$, see Table 1. A disubstituted double bond in the side chain of the base was observed at $\delta_{\mathrm{C}} 129.5$ and 130 . The $E$ geometry for the double bond was supported from the chemical shift of the allylic carbons at $\delta_{\mathrm{C}} 32.3$ and $34.9\left(Z\right.$ geometry $\left.=\delta_{\mathrm{C}} 27.0\right)$.

HPLC analysis of the methylated sugar moiety indicated the sugar moiety to be glucose (glucose $t_{\mathrm{R}}=14.11 \mathrm{~min}$, galactose $t_{\mathrm{R}}=13.27 \mathrm{~min}$ ). The coupling constant of the anomeric proton at $\delta_{\mathrm{H}} 4.93(1 \mathrm{H}, \mathrm{d}, J=8.0 \mathrm{~Hz})$ and the chemical shift of the anomeric carbon $\delta_{\mathrm{C}}$ (104.9) confirmed the $\beta$-configuration of the glucopyranoside moiety ( $\alpha$-glucopyranoside: $\left.J=3.7 \mathrm{~Hz} ; \delta_{\mathrm{C}} 98.5\right) .{ }^{18}$ The absolute configuration of the sugar moiety was determined using the Tanaka et al. method. ${ }^{18}$ HPLC analysis of the reaction mixture exhibited a peak at $t_{\mathrm{R}}=18.5 \mathrm{~min}$, which were coincided with the arylisothiocyanate derivative of $\mathrm{D}$-glucose, confirmed the absolute configuration of the sugar moiety (L-glucose $t_{\mathrm{R}}=19.22$ $\min )$.

In the positive FAB-MS spectral of TSC-2, a series of molecular ion peaks due to $810,824,838,852,866$ and 880 [M $+\mathrm{Na}]^{+}$were observed. Therefore, TSC-2 is presumed to be a molecular species consisting of a phytosphingosine-type cerebroside possessing mainly a 2-hydroxy fatty acid moiety (normal methyls at $\delta_{\mathrm{C}} 13.6$ ) and a $\beta$-D-glucopyranose moiety.

The core structure of the phytosphingosine skeleton in TSC-2 was characterized by comparison of its ${ }^{1} \mathrm{H}$ and ${ }^{13} \mathrm{C}$ NMR spectral data (Fig. 1 and Table 1) with that of known cerebrosides. ${ }^{28}$ The relative stereochemistry of the ceramide moiety is presumed to be $\left(2 S, 3 R, 4 R, 2^{\prime} R\right)$ since the characteristic ${ }^{13} \mathrm{C}$ NMR signals (C-1, 2, 3, 4, $1^{\prime}$ and $\left.2^{\prime}\right)$ and the optical rotation value $\left([\alpha]_{\mathrm{D}}^{22}=+29.4\right)$ are in good agreement with those of the 
phytosphingosines-type glucocerebroside molecular species possessing a $2 S, 3 R, 4 R, 2^{\prime} R$ configuration. ${ }^{28}$

In the FAB-MS analyses of the methanolysis products of TSC-2, the FAME mixture showed molecular ion peaks at 343, 357, 371, 383 and $413[\mathrm{M}+\mathrm{H}]^{+}$, indicating the presence of C-20-C-25 fatty acid methyl esters, possessing normal terminal methyl groups $\left(\delta_{\mathrm{C}}\right.$ 13.6). The LCB also indicated the presence of a C-16 and C-18 long chain base identified from the corresponding molecular ion peaks at of the LCB acetates at $m / z 455[\mathrm{M}+\mathrm{H}]^{+}$and $484[\mathrm{M}+\mathrm{H}]^{+}$ respectively. See Fig. 1 and Table 1.

\subsection{Discussion}

A sphingosine-type (TSC-1) and phytosphingosine-type (TSC-2) cerebrosides, with both containing mainly a 2-hydroxy fatty acids and $\beta$-D-glucopyranose moieties were isolated from the $n$ hexane fraction of $T$. sanguinea in this study. Cerebrosides and ceramides have received a lot of interest in their isolation and characterization due to their significant biological activities such as immunomodulatory antioxidant, antitumour, antiinflammatory and antiviral. ${ }^{29,30}$

Seven triterpenes were isolated from the $n$-hexane fraction of plant: $\beta$-sitosteryl-3 $\beta$-D-glucopyranoside- $O$-fatty acid methyl esters molecular species TSS-1, $\beta$-sitosterol-3 $\beta$-D-glucopyranoside-6'-O-palmitate 1, $\beta$-sitosterol $2, \beta$-sitosterol-3 $\beta$-D-glucopyranoside $\quad 3, \quad \beta$-stigmasterol $\quad 4, \quad \beta$-stigmasterol-3 $\beta$-Dglucopyranoside 5, cholesterol 6 and betulinic acid 7. Biological functions of plant sterols include antihelmintic, antidiabetic, antiinflammatory, antiapoptotic, antinociceptive, antioxidant, immunomodulatory and neuroprotective in neurodegenerative disorders like Alzheimer's disease. ${ }^{31,32}$

Betulinic acid exhibits a variety of biological and medicinal properties such as inhibition of HIV, antibacterial, antimalarial, antiinflammatory, antihelmintic, antinociceptive, anti-HSV-1 and anticancer activities. ${ }^{33}$

The five lignans isolated from the ethyl acetate fraction of $T$. sanguinea: (+)-epipinoresinol 8, (+)-pinoresinol 9, (+)-cycloolivil 10, $(+)$-secoisolariciresinol 11 and $(+)$-isolariciresinol 12 are reported to also have antiviral, antifungal, antimicrobial antifeedant and insecticidal properties and are probably related to plant defense against various pathogens and pests. They also have significant biological activities including antitumour, antiinflammatory, immunosuppression, cardiovascular, antioxidant and antiviral. ${ }^{34}$ Pinoresinol and secoisolariciresinol are mammalian lignan precursors which are converted into enterodiol (END) and enterolactone (ENL) by the intestinal microflora. ${ }^{22,35}$ These enterolignans afford protection against osteoporosis, cardiovascular diseases, liver diseases, hyperlipidemia, breast cancer, colon cancer, prostate cancer and menopausal syndrome. ${ }^{25,36,37}$ The flavanone (+)-eriodictyol 13, also isolated from the ethyl acetate fraction is reported to have significant antiinflammatory, anticancer, neurotrophic, and antioxidant effects. ${ }^{38}$

\section{Conclusions}

In summary, this paper describes the isolation of two glucocerebrosides, TSC-1 and TSC-2, one molecular species TSS-1, seven triterpenes 1-7, five lignans 8-12, and one flavanone 13, from the $n$-hexane and ethyl acetate fractions of whole plant of T. sanguinea. To the best of our knowledge, all the isolated compounds from $T$. sanguinea in this study are being reported for the first time. These compounds have a wide range of biological activities and may act individually or in synergy to produce these biological effects. They may therefore be partly, or wholly responsible for these biological actions, giving credence to the use of $T$. sanguinea in traditional medicine. Further work to increase the amount of isolated cerebrosides molecular species to determine the double bond location in the long chain bases as well as purify and characterize the individual pure cerebrosides is underway.

\section{Conflicts of interest}

There are no conflicts to declare.

\section{Acknowledgements}

We are grateful to the Centre for Plant Medicine Research, Akuapem-Mampong, Ghana, for proving the plant material for this study. We are also grateful to Mr M. Inada and Dr N. Tsuda of the Scientific Support Section of Joint Research Center, Nagasaki University, for ${ }^{1} \mathrm{H},{ }^{13} \mathrm{C}$ NMR and MS measurements. This work was supported by Ministry of Science, Culture, Technology and Sports (MEXT), Japan, which is gratefully acknowledged.

\section{References}

1 A. A. Jigam, U. T. Abdulrazaq, H. A. Mahmud and F. O. Tijani, J. Appl. Pharm. Sci., 2012, 2, 47-51.

2 M. A. Gyamfi and Y. Aniya, Hum. Exp. Toxicol., 1998, 17, 418423.

3 J. D. N'guessan, M. R. Dinzedi, N. Guessennd, A. Coulibaly, M. Dosso, A. J. Djaman and F. Guede-Guina, Trop. J. Pharm. Res., 2007, 6, 779-783.

4 F. C. Ohiri and V. C. Uzodinma, Fitoterapia, 2000, 71, 176178.

5 A. V. Kouakou, J. D. N'guessan, A. K. M. Kra and F. GuédeGuina, J. Soc. Ouest-Afr. Chim., 2006, 22, 21-25.

6 J. D. N'guessan, A. P. Bidié, B. N. Lenta, B. Weniger, P. André and F. Guédé-Guina, Afr. J. Biotechnol., 2007, 6, 1685-1689.

7 I. I. Ohtani, N. Gotoh, J. Tanaka, T. Higa, M. A. Gyamfi and Y. Aniya, J. Nat. Prod., 2000, 63, 676-679.

8 C. C. Chang, Y. C. Lien, K. C. S. C. Liu and S. S. Lee, Phytochemistry, 2003, 63, 825-833.

9 M. A. Gyamfi and Y. Aniya, Toxicology, 2001, 164, 171.

10 T.-T. Zhang, L. Yang and J.-G. Jiang, Food Funct., 2015, 6, 2588-2597.

11 M. A. Gyamfi, I. I. Ohtani, E. Shinno and Y. Aniya, Food Chem. Toxicol., 2004, 42, 1401-1408.

12 A. K. Nyarko and M. E. Addy, J. Ethnopharmacol., 1994, 41, 45-51.

13 K. S. Konan, A. Toure, K. Ouattara, A. J. Djaman and J. D. N'guessan, Afr. J. Microbiol. Res., 2012, 6, 6247-6251. 
14 N. L. Etkins, Trop. Doct., 1997, 27, 12-16.

15 M. A. Gyamfi, M. Yonamine and Y. Aniya, Gen. Pharmacol., 1999, 32, 661-667.

16 M. A. Gyamfi, T. Tanaka and Y. Aniya, Life Sci., 2004, 74, 1723-1737.

17 M. A. Gyamfi, N. Hokama, K. Oppong-Boachie and Y. Aniya, Hum. Exp. Toxicol., 2000, 19, 623-631.

18 T. Tanaka, T. Nakashima, K. Ueda, K. Tomii and I. Kouno, Chem. Pharm. Bull., 2007, 55, 899-901.

19 V. A. S. Ng, E. M. G. Agoo, C.-C. Shen and C. Y. Ragasa, Int J Pharm Clin Res., 2015, 7, 643-646.

20 M. Arora and A. N. Kalia, Int. J. Pharm. Pharm. Sci., 2013, 5, 245-249.

21 S. P. Sawan, T. L. James, L. D. Gruenke and J. C. Craig, J. Magn. Reson., 1979, 35, 409-413.

22 A. Ikuta and H. Itokawa, Phytochemistry, 1988, 27, 28132815.

23 E. Okuyama, K. Suzumura and M. Yamazaki, Chem. Pharm. Bull., 1995, 43, 2200-2204.

24 R. Ghogomu-Tih, B. Bodo, B. Nyasse and B. L. Sondengam, Planta Med., 1985, 51, 464.

25 A. H. Banskota, T. Usia, Y. Tezuka, K. Kouda, N. T. Nguyen and S. J. Kadota, J. Nat. Prod., 2002, 65, 1700-1702.

26 L. A. L. da Silva, L. G. Faqueti, F. H. Reginatto, A. D. C. dos Santos, A. Barison and M. W. Biavatti, Rev. Bras. Farmacogn., 2015, 25, 375-381.
27 R. Higuchi, M. Inagaki, K. Togawa, K. T. Miyamoto and T. Komori, Justus Liebigs Ann. Chem., 1994, 635-658.

28 R. Higuchi, J. X. Jhou, K. Inukai and T. Komori, Liebigs Ann. Chem., 1991, 745-752.

29 K. Yamada, R. Matsubara, M. Kaneko, T. Miyamoto and R. Higuchi, Chem. Pharm. Bull., 2001, 49, 447-452.

30 R. Abdelhameed, M. S. Elgawish, A. Mira, A. K. Ibrahim, S. A. Ahmed, K. Shimizu and K. Yamada, RSC Adv., 2016, 6, 20422-20430.

31 C. Shi, F. Wu, X. Zhu and J. Xu, Biochim. Biophys. Acta, 2013, 1830, 2538-2544.

32 S. Saeidnia, A. Manayi, A. R. Gohari and M. Abdollahi, Eur. J. Med. Plants, 2014, 4, 590-609.

33 G. M. Moghaddam, B. H. A. Faujan and S.-K. Alireza, Pharmacol. Pharm., 2012, 3, 119-123.

34 J.-Y. Pan, S.-L. Chen, M.-H. Yang, J. Wu, J. Sinkkonen and K. Zoud, Nat. Prod. Rep., 2009, 26, 1251-1292.

35 S. Heinonen, T. Nurmi, K. Liukkonen, K. Poutanen, K. Wahala, T. Deyama, S. Nishibe and H. Adlercreutz, J. Agric. Food Chem., 2001, 49, 3178-3186.

36 H. Adlercreutz, Crit. Rev. Clin. Lab. Sci., 2007, 44, 483-525. 37 J. M. Landete, Food Res. Int., 2012, 46, 410-424.

38 S. E. Lee, H. Yang, G. W. Son, H. R. Park, C.-S. Park, Y.-H. Jin and Y. S. Park, Int. J. Mol. Sci., 2015, 16, 14526-14539. 